\title{
Faire de la science au Chili : la recherche par projet comme seul horizon
}

Jeanne Hersant

\section{(2) OpenEdition \\ 1 Journals}

Édition électronique

URL : https://journals.openedition.org/ejts/6602

DOI : $10.4000 /$ ejts. 6602

ISSN : $1773-0546$

Éditeur

EJTS

Référence électronique

Jeanne Hersant, «Faire de la science au Chili : la recherche par projet comme seul horizon », European Journal of Turkish Studies [En ligne], 30 | 2020, mis en ligne le 30 octobre 2020, consulté le 22 mai 2021. URL : http://journals.openedition.org/ejts/6602 ; DOI : https://doi.org/10.4000/ejts.6602

Ce document a été généré automatiquement le 22 mai 2021

(C) Some rights reserved / Creative Commons license 


\title{
Faire de la science au Chili : la recherche par projet comme seul horizon
}

\author{
Jeanne Hersant
}

Jeanne Hersant est professeure assistante du Département de travail social de l'Université pontificale catholique du Chili. Chercheuse titulaire du programme PIA-ANID ANILLO SOC180025 "Women in mathematics in Chile. Sociology of a scientific field from an interdisciplinary and gender perspective".

Chercheuse responsable du projet Fondecyt Regular 1180038 "Justicias reformadas y acceso a la justicia en Chile: sociología del actuar y la recepción judicial (2000-2020)".

Dernière publication : Sommerlad, Hilary; Clarke, Thomas; Gustaffson, ; Hersant, Jeanne ; Holvast, Nina ; Rebecca Sandefur; Verzelloni, Luca, "Paralegals and the casualisation of legal labour markets" in Abel, Rick; Hammerslev, Ole; Schultz, Ulrike; Sommerlad, Hilary (eds), Lawyers in the Twenty First Century, II, Londres, Hart Publishing (à paraître en 2021).

1 Le Chili se caractérise par l'absence d'une politique scientifique proprement dite: certes le pays dispose depuis les années 1960 de nombreux instruments pour financer la recherche (Mullin et al. 2000) mais ceux-ci ne parviennent pas à pallier l'absence d'une action concertée et planifiée (Huerta 2018), dotée d'une institutionnalité propre. Le tout nouveau ministère des Sciences, de la Technologie, de la Connaissance et et de l'Innovation ${ }^{1}$, inauguré en 2019, a succédé à une Commission rattachée depuis 1967 au ministère de l'Éducation ${ }^{2}$. Cette Commission, qui a failli être rattachée au ministère de l'Économie en 2015, fonctionnait surtout comme une agence de moyens et a été incorporée sous un autre nom ${ }^{3}$ au ministère des Sciences au $1^{\text {er }}$ janvier 2020.

2 Le financement de la recherche au Chili - à peine 0,38 \% du PIB national en 2019 (Yañez 2019) contre une moyenne de 2,4 \% dans les pays de l'OCDE (Martínes 2018) - mise 
exclusivement sur la recherche par projet. Cela implique, d'une part, une forte dimension appliquée, notamment dans les disciplines qui touchent aux secteurs stratégiques pour l'économie chilienne - extraction minière, pisciculture et agriculture. D'autre part, les projets financés comportent un budget important dédié aux collaborations internationales, en partant du principe que les chercheur.es du pays ont besoin de construire de solides réseaux de collaboration avec des chercheur.e.s étranger.e.s afin de bénéficier d'un transfert de compétences et de connaissances.

Dans une telle configuration, il est difficile d'installer des lignes et programmes de recherche de long terme, d'autant que le mode d'évaluation des enseignant.e.schercheur.e.s privilégie le productivisme et le court terme et qu'il n'existe pas au Chili une carrière pour les chercheur.e.s en dehors de l'université - à la différence de la France avec le CNRS ou de l'Argentine avec le Conseil national pour la recherche scientifique et technique (CONICET). Le thème du financement de la recherche au Chili est en effet difficilement dissociable de celui des financements des universités : l'État subventionne aussi bien les universités publiques - qui doivent assurer leur autonomie financière - que les universités privées - qui reçoivent parfois bien plus de subventions que les universités publiques. Ce financement se fait en fonction de critères «d'excellence» comme le score des étudiants au concours national d'entrée à l'université (Prueba de Selección Universitaria) et le nombre de publications indexées dans les bases de données Web of Science (Thomson Reuters) et - dans une moindre mesure - Scopus (Elsevier).

4 Ce système de financement et d'évaluation a nécessairement une incidence sur les pratiques de recherche et convertit souvent les activités de recherche en rentes de situation en raison des rémunérations qu'elles impliquent ${ }^{4}$. Je présenterai tout d'abord en détail le fonctionnement du financement de la recherche par projet, du point de vue des chercheur.e.s qui postulent à ces financements (I). Je décrirai ensuite les principes d'évaluation des chercheur.e.s au Chili (II). Je présenterai en conclusion les pratiques et relations asymétriques qui en découlent entre chercheur.e.s consacré.e.s par l'obtention de fonds de recherche, d'une part, chercheur.e.s précaires et assistant.e.s de recherche (qui ont le statut de "personnel technique »), de l'autre. Mon parti pris ici est moins celui d'une analyse externe que d'un témoignage certes étayé et circonstancié, néanmoins subjectif. Le texte qui suit, par conséquent, n'est pas le résultat d'un processus de recherche systématique, et exprime le point de vue de l'autrice - en tant que femme, chercheuse en sciences sociales, en situation de privilège car travaillant dans l'une des universités les plus prestigieuses d'Amérique latine.

\section{Le fonctionnement de la recherche : le financement par projet}

5 La Commission nationale pour la recherche scientifique et technologique (CONICYT) remplacée depuis le $1^{\mathrm{er}}$ janvier 2020 par l'Agence nationale pour la recherche et le développement (ANID) $)^{5}$ - a été créée en 1967 pour centraliser et étatiser les fonds alloués à la recherche, jusqu'alors attribués au sein des principales universités. Tous les financements de la recherche, depuis les gros financements pluriannuels jusqu'aux allocations de master et doctorat en passant par les contrats postdoctoraux (depuis 2002) sont attribués dans le cadre de concours nationaux. Les principaux concours qui mobilisent les chercheur.e.s chaque année - Fondecyt Regular pour les chercheur.e.s 
confirmé.e.s et Fondecyt Iniciación pour les jeunes chercheur.e.s (jusqu'à 7 ans après le doctorat, plus pour les femmes ayant eu des enfants) existent depuis 1981 et 2006 respectivement.

Leur taux de sélection des projets est d'environ $30 \%$ aujourd'hui. Il est certes bien plus élevé que dans d'autres pays comme les États-Unis et la France, mais il masque de profondes disparités. Environ $60 \%$ des projets financés sont mis en œuvre dans la capitale, notamment dans les deux plus grosses universités du pays que sont l'Université du Chili et l'Université Catholique du Chili. Toutes les disciplines entrent en compétition dans ces appels à projet, sans qu'il y ait de critère explicite qui explique les disparités de financement entre les disciplines ${ }^{6}$. Enfin, outre les disparités régionales, les inégalités de genre sont fortes dans ces projets qui ont souvent un.e porteur.e et unique chercheur.e. Entre 2007 et 2016, le taux de projets sélectionnés portés par une femme est passé de $32 \%$ à $38 \%$. Mais selon les disciplines, la brèche - selon l'expression utilisée en espagnol - est plus ou moins cruelle : en 2019, pour la première fois depuis 30 ans, en mathématiques aucune femme ne s'est vue attribuer un financement « Fondecyt Regular »; à titre de comparaison pour la période 2013-2016 seuls 13,5\% des fonds Fondecyt Regular ont été attribués à des projets portés par une femme (Cortez \& Hersant 2016 : 68). L'obtention d'un de ces financements est pourtant un atout certain pour être recruté 8 , et surtout un jalon indispensable dans l'avancement de carrière de tout.e chercheur.e, puisqu'ils fonctionnent comme une instance de validation au sein de son institution d'appartenance et au sein de sa discipline (Maillet 2016).

7 Ces deux principaux types de financement - Fondecyt Iniciación et Regular - financent un chercheur et une petite équipe jusqu'à environ 20000 euros par an (Fondecyt Iniciación) et 60000 euros par an (Fondecyt Regular) - le montant alloué varie d'un projet à l'autre selon ses caractéristiques et l'évaluation qui en est faite par l'agence de la recherche - pour des périodes de deux à quatre ans. De ces sommes, environ 7000 euros bruts annuels -6250 euros nets selon le régime fiscal en vigueur pour les auto-entrepreneurs ${ }^{9}$ - sont attribués de plein droit au ou à la porteur.e du projet comme "prime", plus 7000 euros bruts à répartir entre les chercheur.e.s qui composent son équipe (dans le cas de Fondecyt Regular). Étant donné le relativement bas niveau des salaires dans la plupart des universités, notamment de province, cette prime annuelle équivaut à deux ou trois mois de salaire du ou de la porteur.e de projet. Si le montant est attractif, il ne doit cependant pas faire oublier un aspect central de ce mode de financement: les chercheur.es porteur.e.s de projet deviennent débiteurs.trices de l'agence de la recherche dès lors qu'ils ou elles obtiennent un des deux financements mentionnés (c'est le cas également pour les financements postdoctoraux). En effet, avant de recevoir les montants alloués, ils ou elles doivent signer une reconnaissance de dette (pagaré) à l'institution, par laquelle ils s'engagent à rembourser les sommes dont la déclaration n'est pas validée par l'agence. Les fonds sont ensuite déposés sur un compte bancaire personnel et exclusif que les chercheur.e.s gèrent directement, à charge pour eux ou elles de convaincre leur banque d'ouvrir un compte courant à leur nom. Au Chili, il faut postuler auprès de la banque pour avoir un compte courant (l'équivalent d'un compte chèque) et justifier pour cela d'un certain niveau de revenus. Par défaut, de nombreuses personnes ne peuvent prétendre qu'à un compte d'épargne, modalité non admise par l'agence de la recherche.

Pour pouvoir toucher la prime associée au projet financé, les chercheur.e.s doivent se transformer en prestataires de service et facturer ce montant à leur université - selon 
un artifice juridique puisqu'il est théoriquement impossible de facturer une prestation à son employeur et que de toute façon les financements proviennent de l'agence de la recherche. La prestation de service est, de façon générale, le mode de rémunération d'environ $30 \%$ des personnels de la recherche (et en général dans l'administration publique et les collectivités locales), que l'on nomme aussi des "faux salariés " (Stecher ; Sisto 2020) : ils ou elles ont un contrat à durée déterminée avec une entité ou directement avec le ou la porteur.e du projet qui les rémunère directement - à qui ils présentent une note d'honoraires (boleta de honorarios). Ils ou elles doivent payer 10,7 $\%$ des montants perçus à titre d'impôts, et doivent cotiser eux-mêmes auprès de l'assurance-santé et du fonds de retraite. Le statut de prestataire de service associé au paiement par note d'honoraire exonère en effet du paiement des charges sociales par l'employeur - qui théoriquement n'en est pas un puisque cette figure de prestation de service implique qu'il n'existe pas de relation de subordination entre un.e employeur et un.e employé.e. Ce statut est similaire au statut d'auto-entrepreneur tel qu'il a été décrit par Hélène Stevens (2012) ou Sarah Abdelnour (2017).

9 L'agence de la recherche propose également des financements dits « associatifs » - les projets ANILLO ou MILENIO - qui mobilisent des équipes de chercheurs de différentes universités et disciplines, offrent des sommes plus importantes (100 000 euros par an) et font l'objet d'appels à projet spécifiques par aires de connaissance (sciences sociales et humaines, sciences exactes et ingénierie). Il existe aussi des financements dédiés à la recherche appliquée (FONDEF). La plupart de ces fonds sont cumulables. Sans entrer dans le détail, le mécanisme de sélection et d'évaluation est similaire, et la logique sous-jacente est semblable à celle du financement par projet: une prime pour les chercheurs, un pourcentage du financement attribué à l'université du porteur, l'obligation de présenter un ou plusieurs " produits » à l'issue du projet, sous forme d'un ou plusieurs articles (jusqu'à 4) publiés dans des revues indexées à haut facteur d'impact. La différence ici est qu'étant donné l'importance des fonds en jeu, la gestion en est déléguée aux universités - qui ne disposent pas toujours d'unités ad hoc et recourent également fréquemment à la prestation de service.

L'obtention de financements est un passage obligé, quitte à les cumuler - ce à quoi les chercheur.e.s sont souvent contraint.e.s ou incité.e.s car une partie de ces financements (environ $10 \%$ ) est attribuée à leur université, voire directement à leur unité selon le schéma d'organisation et de gestion propre à chaque université. Dans le Département de travail social qui m'emploie, ces fonds sont notamment utilisés pour payer des enseignants vacataires afin d'alléger la charge d'enseignement des collègues qui obtiennent un financement et libérer ainsi du temps de recherche ${ }^{10}$. Dans la première université - privée - où j'ai travaillé, de 2011 à 2013, ces $10 \%$ étaient reversés à chaque chercheur selon la modalité de l'«overhead», une prime non imposable en somme, qui venait s'ajouter aux honoraires prévus par l'agence de la recherche. Désormais, les universités doivent justifier l'usage qu'elles font de ces financements attribués au titre des « frais administratifs ». Dans la seconde université où j'ai travaillé de 2013 à 2018, une université publique, les $10 \%$ rapportés par chaque projet étaient administrés par l'université comme une source de revenus supplémentaire et les chercheurs qui en étaient à l'origine ne pouvaient y prétendre, ne serait-ce que pour renouveler leur chaise de bureau.

11 La modalité de financement interne de la recherche n'a pas disparu: face à la compétitivité croissante des fonds attribués par l'agence de la recherche, nombre 
d'universités disposent de fonds internes - qui n'incluent pas le paiement d'honoraires - mais cette modalité est loin d'avoir le prestige des fonds de l'agence de la recherche, et ne pèse pas du tout de la même façon dans l'évaluation et l'avancement de carrière. Les financements internes sont plutôt conçus comme un tremplin vers un financement externe plus prestigieux, dont l'obtention est cruciale pour l'avancement de carrière des enseignant.e.s chercheur.e.s. Je présenterai maintenant les processus d'évaluation interne et externe, et leur place dans les évolutions de carrière.

\section{La double évaluation de la performance des chercheur.e.s}

Dans son ouvrage sur Les dérives de l'évaluation de la recherche, Yves Gingras (2014) pointait la façon dont les indicateurs bibliométriques se sont ajoutés à des évaluations déjà fort nombreuses pour les enseignant.e.s chercheur.e.s, et comment ces indicateurs ont accompagné un processus d'évaluation de plus en plus présent au sein même des universités, en plus de l'évaluation du travail scientifique par les revues et les instances de financement de la recherche. Au Chili, cette forme d'évaluation de la "performance » des enseignant.e.s chercheur.e.s est tout à fait installée, qui doivent souvent s'engager, en amont, sur un plan annuel d'activités et d'objectifs. La publication d'articles scientifiques dans des revues indexées et la soumission de candidatures à des appels à projets de la recherche en sont les principaux items.

\section{L'évaluation interne des performances annuelles}

L'obtention de financements est un passage obligé, on l'a vu, dans la carrière d'un.e enseignant.e-chercheur.e; il n'est d'ailleurs pas anodin que dans les évaluations de carrière, l'activité "recherche » et l'activité "publications " soient évalués sous des rubriques séparées. Cela n'est pas du tout intuitif pour quelqu'un d'extérieur au contexte chilien, puisque les publications découlent de la recherche. Or, dans l'item " recherche » n'est évaluée que la participation à des projets ou, mieux, la capacité à obtenir un financement en tant que porteur.e de projet. Quant aux publications, elles ne sont évaluées le plus souvent qu'à travers une grille qui attribue un certain nombre de points aux revues selon leur indexation (Web of Science obtenant toujours le maximum de points) et leur facteur d'impact, quand bien même celui-ci n'indique absolument rien de la qualité des articles qui y sont publiés (Gingras 2014; Koch; Vanderstraeten 2019). Ces critères sont souvent calqués sur ceux qu'utilise l'agence chilienne de la recherche dans son évaluation des CV des postulants, et équivalent en pratique à une injonction à publier en anglais ou du moins hors du Chili. Les bases de données bibliométriques hispanophones et latinoaméricaines (Scielo, Latindex) sont peu valorisées : seules 47 revues publiées au Chili sont indexées dans la base de données Web of Science, toutes disciplines confondues (Koch; Vanderstraeten 2019: 724) ${ }^{11}$. En sciences sociales et humaines, elles se comptent sur les doigts d'une main. Cette énorme pression à l'internationalisation et à la publication «indexée » a eu pour résultat d'importantes modifications dans les pratiques de publications des chercheur.e.s du Chili dans les 40 dernières années et la proportion d'auteur.e.s. chilien.ne.s dans la base de données WoS est passée de 0,10 \% en 1976 à 0,34 \% en 2015 (Koch, Vanderstraeten 2019: 727). Cela n'en reste pas moins une injonction fortement 
discriminatoire pour les chercheur.e.s les moins bien doté.e.s en ressources ou capital propre, pour affronter le coût d'une publication en anglais et les délais de publication dans les revues les plus prestigieuses. Celles et ceux qui peuvent se le permettre sont déjà dans une position confortée au sein de leur unité.

Enfin, un effet pervers de ce système dans les universités de second rang du moins - lié à une vision positiviste de la qualité de la recherche mais également, on l'a vu, aux nécessités pour les universités d'obtenir des financements de l'État sur la base du nombre d'articles indexés qu'elles publient - est une course à la publication indexable par WoS, peu importe la qualité de la revue. En effet, l'évaluation des chercheur.e.s n'est qu'une face de la médaille. Afin d'inciter leurs chercheur.e.s à publier dans des revues indexées, la plupart des universités au Chili ont un système de primes pour chaque article publié ${ }^{12}$. Selon les universités, les disciplines et le facteur d'impact ou «quartile » de la revue, la prime versée peut aller de 1500 à près de 3000 euros. Dans les disciplines où l'on publie beaucoup, comme les sciences biologiques, les primes versées aux chercheur.e.s publiant.e.s peuvent donc atteindre des montants considérables, et ne sont pas toujours plafonnées. Enfin, il est impossible d'obtenir un financement de l'agence de la recherche sans un certain nombre d'articles indexés - le nombre dépend du prestige de l'indexation - au cours des cinq années précédentes (pour les femmes, ce délai augmente d'un an par enfant de moins de cinq ans). La boucle est bouclée, et cette course aux publications génère des pratiques peu regardantes envers l'éthique scientifique. Dans l'une des universités où j'ai travaillé, un programme de master incitait explicitement - par courrier électronique - les chercheur.e.s du Centre d'études avancées de l'Université (sans obligation d'enseignement) à diriger des mémoires avec l'argument suivant : la perspective de cosigner un article avec leurs étudiants et de leur faire signer un document par lequel ceux-ci renoncent à leur droit d'auteur, pour pouvoir ainsi toucher la totalité de la prime par article (la prime doit être partagée lorsque l'article est publié par des coauteurs de la même institution). Cette même université a été témoin d'un scandale de plagiat d'un mémoire d'un étudiant de premier cycle par son directeur de mémoire. Deux autres personnes ont été impliquées puis - paradoxalement - blanchies (aussi bien dans le cadre de la procédure interne que devant la justice civile) : dans le cadre d'un arrangement à trois où les auteurs s'invitaient mutuellement dans leur production, ces deux personnes n'avaient fait « que » co-signer l'article plagiaire, sans en être réellement autrices, ni être informées du plagiat.

\section{L'évaluation externe par les pairs pour l'obtention de financements de l'agence de la recherche}

Pour pouvoir avoir une chance d'obtenir un financement de l'agence de la recherche, il faut en premier lieu avoir un CV " compétitif » : l'un des critères de l'évaluation est la viabilité du projet, et le registre de publications compte au moins autant que la solidité du projet. Pour les financements les plus importants, les projets sont soumis et évalués en anglais ; ce n'est pas le cas des projets de type Fondecyt qui doivent être soumis en anglais seulement dans le cas des sciences dites exactes. Pour les sciences sociales et humaines, le projet peut être soumis en anglais ou espagnol, il sera évalué par défaut au Chili à moins que les postulant.e.s ne suggèrent des évaluateurs étrangers. 
16 Le processus d'évaluation organisé par l'agence de la recherche, crucial pour l'obtention d'un financement, est souvent lapidaire et discrétionnaire. En premier lieu, la liste des publications donne aux postulant.e.s un score qui compte jusqu'à $30 \%$ dans la note finale (pour Fondecyt Regular). En Sociologie et Communications, chaque article publié rapporte un certain nombre de points en fonction de l'indexation de la revue et de son facteur d'impact, peu importe la réputation de la revue au sein d'un groupe de spécialistes $^{13}$. À titre d'exemple, une revue comme Sociologie du Travail, qui bénéficie d'un grand prestige dans son domaine, mais a délibérément quitté le portail Elsevier pour l'accès ouvert, ne rapporterait aucun point. En ce qui concerne l'évaluation du projet, il ne s'agit pas d'une évaluation à l'aveugle : le nom et CV des postulant.e.s sont connus, mais l'identité des évaluateurs.trices n'est jamais dévoilée. La grille d'évaluation fait foi, et si les évaluateurs.trices doivent justifier la note qu'ils appliquent à chaque section, ils peuvent le faire en un ou deux paragraphes. Il est plutôt rare, dans ce processus, de recevoir une évaluation circonstanciée. Les règles d'attribution des évaluateurs.trices sont peu transparentes, et ceux ou celles-ci doivent s'engager à la confidentialité au sujet des projets qu'ils évaluent. Les sciences sociales et humaines souffrent indéniablement de leur manque d'internationalisation, et de la petite taille de la communauté scientifique au Chili même. Ce système d'évaluation favorise en effet, ou du moins ne sanctionne pas, les jeux de pouvoir et de réseaux ou les jugements à l'emporte-pièce. L'extrait ci-dessous du post d'un collègue sur les réseaux sociaux - que je reproduis de façon anonyme avec l'accord de celui-ci - illustre ce double problème: d'une part, le manque de spécialistes sur des sujets ou aires «marginaux»; d'autre part, le mécontentement et l'incompréhension qui dominent souvent à la lecture des rapports d'évaluation :

«Troisième projet [...] sur la philosophie arabe médiévale qu'on nous refuse. Cette fois, c'est pire que les précédentes : deux évaluations nous ont attribué le score maximum (les évaluations internationales ${ }^{14}$ apparemment) et une troisième un score vraiment bas. Pourtant, le pire n'est pas cette mauvaise note, plutôt les fautes d'orthographe dans le rapport de l'évaluateur en question, qui écrit «Khun » au lieu de « Kuhn » ou qui se réfère à « Sanchez Nogales » au lieu de Gómez Nogales (le spécialiste espagnol de philosophie arabe) [...]. En plus de tout ça, il argumente que puisque nous ne sommes pas «spécialistes" (peu importe que nous ayons travaillé sur divers aspects de la philosophie arabe depuis 2007 entre groupes de recherche, enseignements et colloques) nous ne pouvons pas mener de recherches sur le sujet. Pire : il en appelle au manque de citations de «spécialistes » sans voir que le projet ne cite peut-être pas ses amis spécialistes mais de nombreux autres (français, italiens, allemands, etc.). L'évaluateur insiste sur le fait que nous n'utilisons pas de sources arabisantes pour nous référer à un certains "Suhravard " (j'imagine qu'il parle de Sohravardi), alors que toute la bibliographie que nous utilisons sur cet auteur est bilingue (arabe-anglais). Pour couronner le tout, il signale un membre de l'équipe comme non spécialiste seulement parce qu'il a obtenu un doctorat en Histoire en Allemagne et pas un doctorat en philosophie arabe. Tout cela est imprésentable ».

17 Au-delà du dépit d'un collègue, cet extrait met en évidence les aspects quantitatifs et parfois discrétionnaires de l'évaluation des projets de recherche. Un projet peut recevoir deux excellentes appréciations et une catastrophique, il sera défavorisé par rapport à un autre projet qui aura reçu des évaluations moins enthousiastes mais moins divergentes : c'est une logique arithmétique qui prévaudra en faisant la moyenne des notes. Les commentaires et rapports d'évaluation sont souvent considérés comme ayant valeur d'illustration : les membres des comités qui coordonnent l'évaluation et 
classent les projets se réservent le droit de couper les commentaires et n'en transmettre qu'une partie aux candidat.e.s. Pour certains financements, comme les bourses de master ou de doctorat, auxquels je participe comme évaluatrice depuis 2016, laisser un commentaire pour justifier la note attribuée à chaque item est devenu obligatoire en 2018, mais ces commentaires ne peuvent excéder un certain nombre de signes et sont proposés de façon prédéfinie aux évaluateurs.trices, sous la forme de phrases toutes faites, afin de "gagner du temps ». Enfin, ce que signale le post cité, est l'importance parfois disproportionnée que prennent les stratégies de citation: elles doivent comporter une dimension scientifique et une dimension politique afin de montrer l'insertion du ou de la postulant.e dans les « bons » réseaux.

\section{Conclusion}

Dans cet article, j'ai mis l'accent sur le financement et la division du travail scientifique; il eut été tout aussi pertinent d'inclure le fonctionnement des comités d'éthique universitaires comme autre pierre de l'édifice du fonctionnement et du financement de la recherche au Chili. En effet, tout projet financé par l'agence de la recherche doit impérativement être validé par un comité d'éthique universitaire. Cette évaluation se fait souvent à travers une grille, non pas de points cette fois, mais plutôt sous la forme d'une « check list ». Or, à l'image des protocoles d'éthique de médecine et biologie, ceux-ci comportent une liste prédéfinie de conditions sous lesquelles les entretiens seront menés, où la recherche sera réalisée, l'échantillon des personnes interviewées et des preuves que les autorisations institutionnelles ont bien été accordées (Dequirez ; Hersant 2013 : 647).

Enfin, nul besoin d'être spécialiste en théorie du don pour saisir intuitivement le type de relations de subordination et d'obligations qui se nouent autour de ces projets de recherche, dans un contexte où la majorité des chercheur.e.s connaissent la précarité professionnelle en travaillant comme prestataire de services, sans contrat stable. Les prestigieux Fondecyt Regular, Anillo, Milenio, convertissent les chercheur.e.s en chercheur.e.s confirmé.e.s aux yeux de leurs pairs, mais aussi et surtout en entrepreneurs de projets et pourvoyeurs de ressources pour les collègues qu'ils invitent à y participer et pour les assistants de recherche qu'ils recrutent. Le travail du "personnel technique » en particulier est très peu régulé, et il n'y a pas de rémunération minimum garantie. Ce mode de financement de la recherche contribue sans nul doute à la permanence d'une division du travail très marquée dans les sciences sociales chiliennes, où les chercheur.e.s se consacrent à la gestion de projet (postuler et gérer ces projets occupe un temps considérable) et à la tâche noble de mettre en forme des notes de terrain, en les articulant au cadre théorique, pour rédiger des articles. Le travail de terrain et la collecte et analyse de données est encore trop souvent considérée comme une tâche subalterne qui doit être déléguée - le "sale boulot » ou « dirty work » utilement conceptualisé par Everett Hugues (1971).

S'il y a des enseignements à tirer de la situation de la science au Chili, commençons par reconnaître avec Antoine Maillet (2016) la grande vertu de concours ouverts et horizontaux pour obtenir des bourses (de master et doctorat, pour étudier au Chili ou à l'étranger) et financements à tous les stades de la trajectoire universitaire. Ce système de financement - qui a notamment permis à des milliers de Chilien.ne.s de réaliser un doctorat à l'étranger - a sans conteste contribué à enrichir et professionnaliser les 
sciences sociales, dont le paysage aujourd'hui est beaucoup plus varié qu'il ne l'était à mon arrivée dans le pays en 2011. Cela étant, l'exclusivité donnée à la recherche par projet, ainsi que le fonctionnement du financement et de l'évaluation de la recherche tels que je les ai exposés ici mettent en évidence les risques que comportent l'importation en France d'une telle logique pour l'organisation sociale des universités et le processus de production des connaissances, dont la richesse repose justement sur la reconnaissance du temps long dans la mise en place des dynamiques propres à la recherche scientifique.

\section{BIBLIOGRAPHIE}

Abdelnour, Sarah (2017). Moi, petite entreprise : les auto-entrepreneurs, de l'utopie à la réalité, Paris, PUF.

Cortez, María Isabel ; Hersant, Jeanne (2016). "Femmes et mathématiques au Chili”, Synergie Chili, 12, pp. 59-71.

Dequirez, Gaëlle ; Hersant, Jeanne (2013). “The virtues of improvisation: ethnography without an ethics protocol", Current Sociology 61 (5-6), pp. 446-460.

Gingras, Yves (2014). Les dérives de l'évaluation de la recherche. Du bon usage de la bibliométrie, Paris, Raisons d'Agir.

Huerta, Luis (2018). “Una política científica para Chile, jahora!", El Mostrador, 20.04.18. URL: https://www.elmostrador.cl/noticias/opinion/2018/04/20/una-politica-cientifica-para-chileahora/

Hughes, Everett (1971). The sociological eye: selected papers, New Brunswick: Transaction Books.

Koch, Tomás; Vanderstraeten, Raf (2019). "Internationalizing a national scientific community? Changes in publication and citation practices in Chile, 1976-2015", Current Sociology 67 (5), pp. 723-741.

Maillet, Antoine (2016). “¿Politización de Fondecyt ? Un poco de seriedad por favor”, El Mostrador, 18.03.2016. URL: https://www.elmostrador.cl/noticias/pais/2016/03/18/politizacionde-fondecyt-un-poco-de-seriedad-por-favor/

Martínes, Francisco (2018). “AA dónde vamos Chile?”, Revista ¿Qué pasa?, 26.10.2018. URL: https://www.latercera.com/que-pasa/noticia/a-donde-vamos-chile/377075/

Mullin, J., Adam, R., Halliwell, J., Milligan, L. (2000). Science, Technology and Innovation and Chile, Ottawa: International Development Research Centre.

Stecher, Alejandro; Sisto, Vicente (2020). “Trabajo y precarización laboral en el Chile neoliberal. Apuntes para comprender el estallido social de octubre del 2019", in Araujo, Kathya (ed), Hilos tensados: para leer el Octubre chileno, Santiago, Editorial de la Universidad de Santiago de Chile, pp. 37-82.

Stevens, Hélène (2012). « Le régime de l'auto-entrepreneur, une alternative désirable au salariat? », Savoir/Agir, 3 (21), pp. 21-28. 
Yañez, Cecilia (2019). “Ministro de Ciencia y financiamiento científico en Chile: 'La discusión sobre el 0,38\% del PIB me parece obsoleta”, Revista ¿Qué pasa?, 26.04.2019. URL: https:// www.latercera.com/que-pasa/noticia/ministro-ciencia-financiamiento-cientifico-chile-ladiscusion-038-del-pib-me-parece-absurda/631724/

\section{NOTES}

1. Ministerio de Ciencia, Tecnología, Conocimiento e Innovación. Voir "Le Chili se dote pour la première fois d'un ministère des Sciences", Courrier International, 08.06.2018. URL: https:// www.courrierinternational.com/article/le-chili-se-dote-pour-la-premiere-fois-dun-ministeredes-sciences

2. Comisión Nacional de Investigación Científica y Tecnológica (CONICYT).

3. Agencia Nacional de Investigación y Desarrollo (ANID).

4. Dans un souci d'honnêteté, il convient de préciser que l'autrice de ces lignes se trouve ellemême dans cette situation puisqu'elle cumule les honoraires associés à deux projets de recherche dont elle est porteuse pour la période 2018-2021. Ces honoraires totalisent environ 11000 euros bruts annuels (le cumul est autorisé jusqu'à 12000 euros bruts annuels); cela correspond à environ 5 mois de salaire additionnels étant donné que je travaille dans un département de travail social, où les salaires sont parmi les plus bas dans mon université. Cela ne correspond qu'à 3 mois de salaire, selon le stade de sa carrière, d'un enseignant chercheur d'une faculté d'Ingénierie. Mais ce n'est bien sûr qu'une estimation puisque les universités privées ne rendent pas publiques leurs grilles de salaires. Il n'y a pas de statut national pour les enseignant.e.s chercheur.e.s : chaque université définit l'échelon et la grille de salaires de ses enseignant.e.schercheur.e.s. Seules les universités publiques, comme le reste de l'administration, ont l'obligation de rendre publiques les rémunérations de leurs fonctionnaires. Dans les universités privées, la grille est modulable selon les facultés et leur « valeur » sur le marché du travail.

5. Dans la suite du texte, j'utiliserai le terme générique d'agence de la recherche, puisqu'en l'occurrence le changement de dénomination ne correspond pas à un changement de politique publique et les modalités d'attribution des financements de recherche sont restées inchangées. 6. Que l'on peut visualiser ici pour la période 2014-19 à travers cette infographie qui représente la répartition des financements par année, discipline, université et en fonction des montants alloués : https://app.powerbi.com/view? r=eyJrIjoiMWEwMDUwODUtNGZlYi00M2IyLWJjY2YtMjEzYTczOGM5Yjg3IiwidCI6ImU3M2FmMWRILWU5ZTYtNGM0OS1iMWUxLWZjNjg3 7. CONICYT, Participación femenina en programas de Conicyt 2007-2016. Departamento de Estudios y Gestión Estratégica, 2017, p. 19. URL: https://app.powerbi.com/view? r=eyJrIjoiMWEwMDUwODUtNGZlYi00M2IyLWJjY2YtMjEzYTczOGM5Yjg3IiwidCI6ImU3M2FmMWRILWU5ZTYtNGM0OS1iMWUxLWZjNjg

8. Il faut théoriquement être en poste pour obtenir ces financements, puisqu'ils requièrent le parrainage d'une institution. Mais il est de plus en plus fréquent, au moins depuis 2012, que les universités conditionnent une embauche - ne serait-ce qu'à mi-temps - à l'obtention d'un Fondecyt Iniciación, voire qu'elles ne s'engagent à rien d'autre qu'au parrainage du ou de la candidate, ce qui est un détournement de ces appels à projet puisque les universités reçoivent un pourcentage du financement du projet au titre des frais d'administration ou gestion que ceux-ci peuvent induire.

9. Je reviens plus bas sur la figure de la «prestation de services».

10. Le service d'enseignement est souvent modulable en fonction des heures de recherche, attribuées en fonction du nombre de projets financés par l'agence de la recherche dont disposent les chercheur.e.s. 
11. Il est impossible de connaitre précisément le nombre de revues au Chili, car elles ne sont pas toutes référencées. La base de données Scielo en dénombre plus de 130, toutes disciplines confondues : https://scielo.conicyt.cl/scielo.php?lng=es\&script=sci_subject

12. À l'exception de l'Université du Chili et de l'Université catholique du Chili. Dans cette dernière, il existe un système de prime à la productivité scientifique, pour un montant bien inférieur (environ 500 euros), à laquelle les chercheur.e.s peuvent prétendre tous les deux ans.

13. En mathématiques, en revanche, les revues sont classées sur le mode du classement du HCERES français, à la différence que le classement inclut les revues de différents pays.

14. Dans l'idiosyncrasie de la recherche au Chili, l'adjectif « international » se réfère à tout ce qui est extérieur au Chili dans les activités scientifiques et de valorisation de la recherche. Un séminaire «international » est un séminaire qui comporte au moins un.e intervenant.e d'une université étrangère.

\section{AUTEUR \\ JEANNE HERSANT \\ Université catholique du Chili \\ jeanne.hersant@uc.cl}

